\title{
Probe-based confocal laser endomicroscopy in the differential diagnosis of inflammatory bowel diseases: a case series
}

\author{
Jung Won Park, Tae Il Kim, Jae Hee Cheon \\ Department of Internal Medicine and Institute of Gastroenterology, Yonsei University College of Medicine, Seoul, Korea
}

Since its introduction in 2004, confocal laser endomicroscopy (CLE) has emerged as a significant imaging tool for gastrointestinal endoscopic examinations. CLE is a technique that allows real-time in vivo microscopic imaging of the gastrointestinal mucosal layer. ${ }^{1}$ The CLE can obtain very high magnification and resolution images using the "confocal" method. This endomicroscopy technique enables endoscopists to obtain real-time in vivo histology data during an ongoing endoscopy procedure, thereby creating processes defined as "optical biopsies."

Previously, diagnosis of IBD including CD and UC, in addition to intestinal Behçet's disease, was primarily based on colonoscopic, pathological, and serological findings. ${ }^{2-4}$ Probe-based CLE (pCLE) may be beneficial in the diagnosis of IBD by allowing microscopic imaging; especially in some cases with macroscopic mucosal healing, clinically relevant inflammatory changes can be visualized with CLE during colonoscopy. ${ }^{5-7}$ However, previous studies have mostly focused on the use of CLE in surveillance of cancer development for IBD patients to detect intraepithelial dysplasia, and most of these studies regarding the CLE have focused on UC and not CD. ${ }^{5,8}$ Intestinal Behçet's disease and intestinal tuberculosis are also known to be quite difficult to distinguish from CD using conventional white light endoscopy, and for this reason, the pathologic findings from biopsy of the lesion

Received March 13, 2018. Revised April 10, 2018.

Accepted April 13, 2018.

Correspondence to Jae Hee Cheon, Department of Internal Medicine,

Yonsei University College of Medicine, 50-1 Yonsei-ro, Seodaemun-gu,

Seoul 03722, Korea. Tel: +82-2-2228-1990, Fax: +82-2-393-6884, E-mail:

geniushee@yuhs.ac contribute substantially to the differential diagnosis. pCLE might represent a potential solution and may serve as an additional diagnostic tool for determining a correct diagnosis of IBD.

The efficacy of pCLE in the differential diagnosis of IBD at an early stage has not been studied to date. Therefore, this study was designed to investigate the concordance of clinical diagnosis and pCLE findings in the differential diagnosis of a patient presenting with ileocecal ulcer and inflammation. Moreover, we compared biopsy results obtained from pCLE findings of the inflamed lesions to evaluate the effectiveness of targeted biopsies in the diagnosis of IBD. This pilot study includes 6 IBD patients who were subjected to pCLE performed by a single gastrointestinal specialist and endoscopist (J.H.C) at the Severance Hospital, Seoul, South Korea.

Two UC patients were included in the study, patients A and B, a 45-year-old man and a 48-year-old woman, respectively. Patient A had been diagnosed with UC 1-year prior and was on maintenance treatment with balsalazide disodium, dehydrate $4.5 \mathrm{~g} /$ day. He tested negative for perinuclear antineutrophil cytoplasmic antibodies (p-ANCA) initially, and his CRP level was $0.5 \mathrm{mg} / \mathrm{L}$ both initially and at the time of the pCLE procedure. Colonoscopy with pCLE was performed and the colon was defined to be in the remission state; however, there were scar changes and a mild stricture at the ascending colon and hepatic flexure. The pCLE of the main lesion in the ascending colon showed widening of the lamina propria, inflammatory infiltrates, goblet cell depletion, and crypt distortion (Fig. 1A and B). The biopsy showed chronic nonspecific inflammation. pCLE was also performed at the normal colon mucosa of the sigmoid colon,

๑ Copyright 2018. Korean Association for the Study of Intestinal Diseases. All rights reserved.

This is an Open Access article distributed under the terms of the Creative Commons Attribution Non-Commercial License (http://creativecommons.org/licenses/by-nc/4.0)

which permits unrestricted non-commercial use, distribution, and reproduction in any medium, provided the original work is properly cited. 

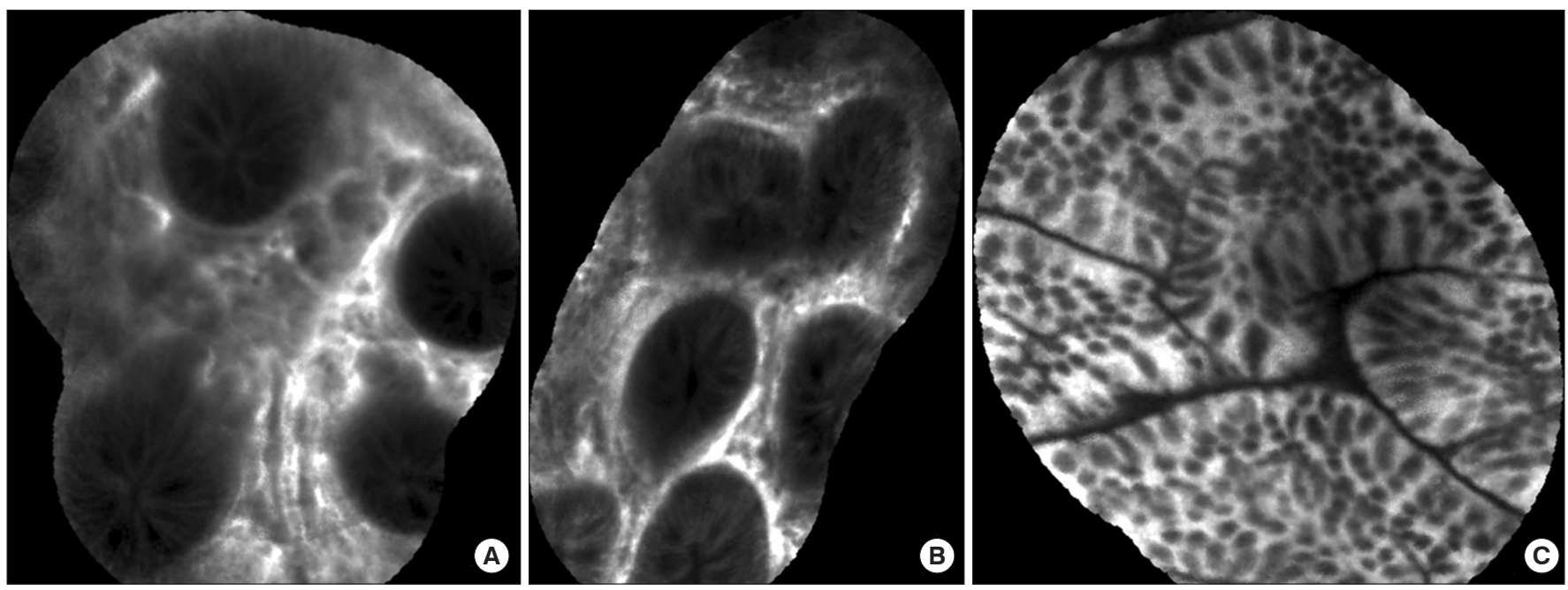

Fig. 1. Changes of the intestinal mucosa detected by probe-based confocal laser endomicroscopy in UC (Patient A). (A) Irregular architecture and increased distance between the colonic crypts. (B) Leakage of fluorescein into the crypt lumen from the interstitium of the colon. (C) Normal colonic crypts with no fluorescein in the lumen and no crypt distortion.

and this showed crypts disposed as straight structures oriented to muscularis mucosae (Fig. 1C). Patient B had been diagnosed with UC, consisting of proctitis only, in 2001, and the disease progressed to pancolitis in 2005. She achieved remission in 2011 with mesalazine $2.2 \mathrm{~g}$ /day orally and mesalazine enema. However, her symptoms worsened in 2013 and the immunomodulator, azathioprine $25 \mathrm{mg}$ daily was administrated. Azathioprine was stopped the following year, because the patient developed neutropenia. p-ANCA testing was negative and the CRP level was $0.4 \mathrm{mg} / \mathrm{L}$ at the time of pCLE. Colonoscopy with pCLE findings indicated the colon in remission, and the UC lesion in the sigmoid colon and rectum showed mild crypt distortion, but no dysplastic cells. The biopsy of this lesion showed chronic nonspecific inflammation.

The next 2 patients were diagnosed with $\mathrm{CD}$, and had of disease durations of 9 and 11 years, respectively. Patient $\mathrm{C}$ was a 44-year-old man who was diagnosed with CD in 2006 but was not quite compliant to the treatment provided in the first clinic and had been under no medical care. He presented at our facility in 2013 with severe abdominal pain. He had undergone small bowel resection owing to partial obstruction of the small bowel and a mesenteric abscess. Anti-Saccharomyces cerevisiae antibody (ASCA) IgG testing showed a positive result, and the CRP level was $76.90 \mathrm{mg} / \mathrm{L}$. After surgery, the patient was maintained on mesalazine $3 \mathrm{~g}$ /day and $50 \mathrm{mg}$ azathioprine maintenance therapy, with the symptoms being controlled and the CRP level reducing to $1.1 \mathrm{mg} /$ L. Colonoscopy with pCLE was performed and revealed a stricture at the ileocecal valve orifice and shallow ulcerations at the ileocecal valve and terminal ileum. pCLE of this main lesion in the terminal ileum and cecum revealed crypt destruction and fluorescent spillage, but a granuloma was not visible on pCLE (Fig. 2A and B). The pathology report of this lesion indicated erosion with chronic nonspecific inflammation. The normal mucosa of the sigmoid colon visualized by pCLE reveled crypts with a straight appearance, with luminal opening of the crypts appearing like black holes (Fig. 2C). Patient D was a 29-year-old man diagnosed with CD in 2004 and was on mesalazine $2 \mathrm{~g}$ /day and $100 \mathrm{mg}$ of azathioprine maintenance therapy. ASCA level was equivocal, and his CRP level at the time of pCLE was $3.1 \mathrm{mg} / \mathrm{L}$. His colonoscopy showed multiple, scattered small aphthous ulcerations from the terminal ileum to the rectum, mostly located at the sigmoid colon and rectum with sparing of normal mucosa showing skipped lesions. pCLE of these lesions also showed crypt destruction and fluorescent spillage, but granuloma was not noticeable. Biopsy of both the terminal ileum and the sigmoid colon showed erosions with chronic nonspecific inflammation in both samples.

Patient E, a 59-year-old man, was diagnosed with intestinal Behçet's disease in 2002; he had undergone a total of 7 intestinal resections, including right hemicolectomy and segmental resection of the transverse colon owing to the recurrence of intestinal Behçet's ulcer. He was on daily $4 \mathrm{~g}$ of mesalazine and was steroid-dependent. CRP level was $12.7 \mathrm{mg} / \mathrm{L}$ at the time of pCLE. His colonoscopy showed huge and deep ulceration at the anastomosis site, located at the ascending colon. pCLE of the ulcer lesion revealed severe inflammatory 

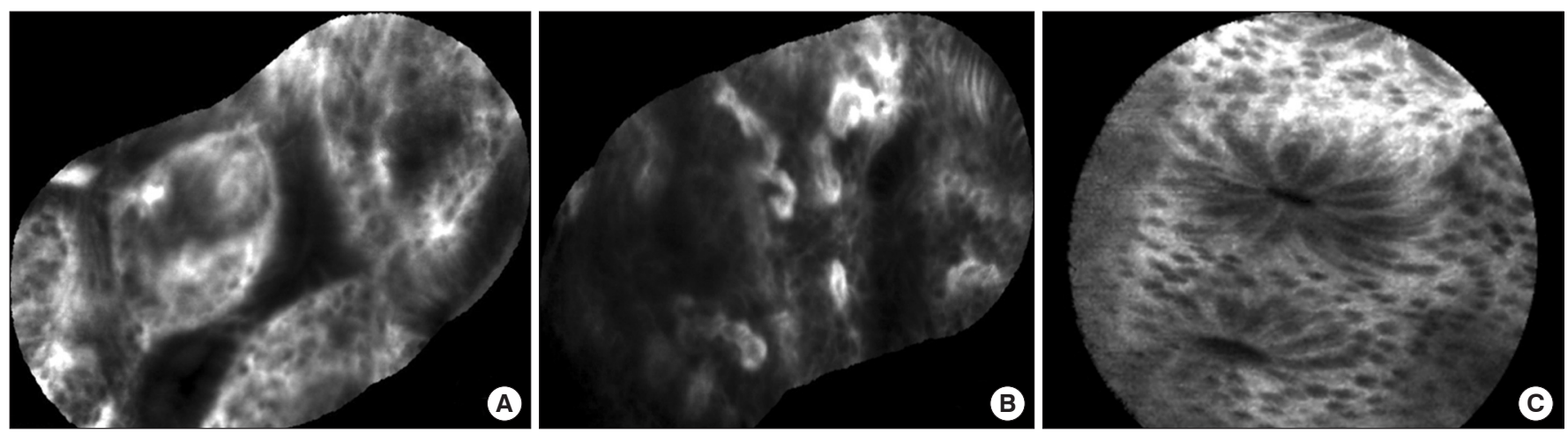

Fig. 2. Changes of the intestinal mucosa detected by probe-based confocal laser endomicroscopy in a CD (Patient C). (A, B) lleocecal valve ulcer; crypt destruction, fluorescent spillage, no granuloma visible. (C) Straight appearance of crypts with luminal opening of the crypts resembling as black holes in the normal mucosa of sigmoid colon.
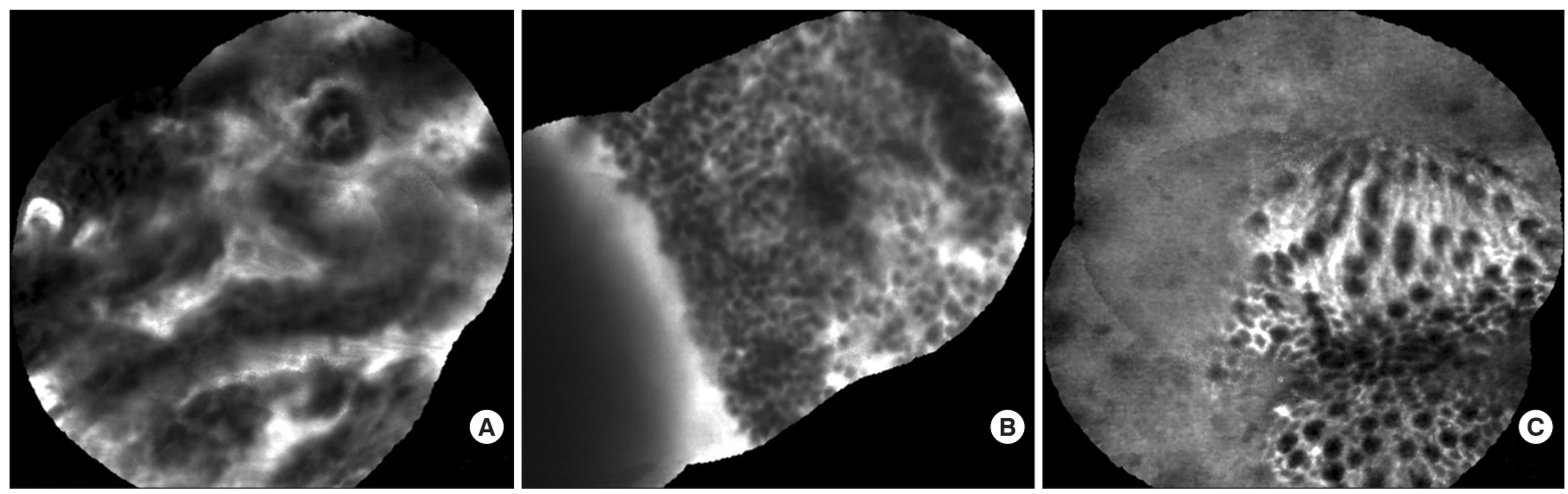

Fig. 3. Changes of the intestinal mucosa detected by probe-based confocal laser endomicroscopy in intestinal Behçet's disease (Patient E). (A) Anastomosis site ulcer; crypt destruction with disarrayed feature. (B) Abundant inflammatory cell infiltration. Granuloma, AFB, or vasculitis not visible. (C) Normal colonic crypts with no fluorescein in the lumen and no crypt distortion.

cell infiltration and crypt destruction but granuloma, $\mathrm{AFB}$, or vasculitis could not be observed (Fig. 3A and B). The biopsy taken from this lesion showed inflamed granulation tissue with ulceration. The normal colon mucosa of the ascending colon was also visualized and revealed normal colonic crypts with no fluorescein in the lumen and no crypt distortion (Fig. 3C).

Patient F, a 46-year-old man, was referred to our clinic for further evaluation of ulcerations located at the terminal ileum and ileocecal valve observed at an external clinic study. Culture and PCR for Mycobacterium tuberculosis showed negative results, with negative biopsy results. The diagnosis of IBD was indeterminate, and pCLE was performed. pCLE of the ulcer site showed crypt destruction, but no granuloma, vasculitis, or AFB were found. The biopsy of this lesion showed chronic nonspecific inflammation.

In this study, we analyzed the pCLE findings of 2 UC patients and 2 CD patients, along with 1 Behçet's disease patient and 1 patient with terminal ileal ulcer due to an unknown cause. The study aimed to evaluate the efficacy of pCLE for the precise diagnosis of IBD. Confocal images were obtained using fluorescein as the fluorescent contrast agent. Allergic reactions to fluorescein, cardiovascular complications, nausea, et cetera, have been reported, but they occur infrequently. No adverse reactions occurred in this study.

Although several studies have reported that changes observed on CLE procedures can predict long-term remission in IBD, those studies were not focused on the initial diagnosis of IBD.$^{5,8} \mathrm{~A}$ precise diagnosis of IBD patient is rather significant, as the treatment of each disease may lead to aggravation of one another. Currently, the diagnosis depends on a comprehensive conclusion derived from endoscopic findings and pathological, culture, and serological test results. Nevertheless, a $20 \%$ chance of misdiagnosis exists 
and eventually the patient might have to undergo surgical intervention for accurate diagnosis and treatment. ${ }^{9}$ For these reasons, adequate tools assisting the differential diagnosis of IBD are desperately needed.

Endomicroscopy is an emerging technology that provides a detailed microscopic analysis of the mucosal surface. Histopathological assessment of IBD biopsies is meaningful for diagnosis. The guidelines for IBD management published by the and European Colitis and Crohn's Organization underline the essential role of pathologic examining for the diagnosis and definition of the type of IBD. ${ }^{3,10}$ In this context, endomicroscopy can be extremely advantageous, with regard to simultaneously determining the location at which to take a biopsy sample and providing an indication of the IBD type during the procedure. However, an important disadvantage of using CLE is the longer procedural time. Extended duration of colonoscopy is uncomfortable for patients and expensive for endoscopy wards. The price of the equipment adds to the increased cost of performing a CLE procedure. Consequently, cost-benefit studies are needed to analyze whether the advantages of CLE can overcome the increased costs of the procedure.

In our study, pCLE of UC lesions showed infiltration of inflammatory cells and widening of the lamina propria, crypt distortion, and destruction due to inflammation. These findings were consistent with the diagnoses of UC and thus may be useful for confirming a suspected diagnosis. However, in $\mathrm{CD}$ and intestinal Behçet's disease, pCLE could recognize the inflammatory cells and mucosal shedding but could not identify any granuloma, AFB, or vasculitis, which are significant specific findings for diagnosis. This leads to the conclusion that pCLE is of little use in the differential diagnosis of these diseases. Unfortunately, no intestinal tuberculosis patients were enrolled; hence, locating AFB with pCLE was not possible in this study.

In conclusion, pCLE provides information on the lamina propria and crypt distortion and destruction due to inflammation and thus may represent a useful tool for the diagnosis of UC. However, there are clear limitations to its use in CD and intestinal $\mathrm{BD}$, as the presence of vasculitis or granuloma are not adequately visualized. Determining the exact efficacy of pCLE in the differential diagnosis of IBD requires further study, including studies in a larger cohort of IBD patients. Moreover, pCLE findings of intestinal tuberculosis patients, which were insufficient in this study, should necessarily be supplemented in future studies.

\section{FINANCIAL SUPPORT}

The authors received no financial support for the research, authorship, and/or publication of this article.

\section{CONFLICT OF INTEREST}

No potential conflict of interest relevant to this article was reported.

\section{AUTHOR CONTRIBUTION}

Conceptualization: JH Cheon. Methodology: JH Cheon, TI Kim. Formal analysis: JH Cheon, TI Kim. Project administration: JW Park, JH Cheon. Visualization: JH Cheon. Writing - original draft: JW Park. Writing - review and editing: JH Cheon. Approval of final manuscript: all authors.

\section{ACKNOWLEDGEMENTS}

We authors thank the 6 patients who participated in this study, who gladly provided their medical records and endoscopy images with huge understanding.

\section{REFERENCES}

1. Kiesslich R, Goetz M, Vieth M, Galle PR, Neurath MF. Confocal laser endomicroscopy. Gastrointest Endosc Clin N Am 2005; 15:715-731.

2. Cheon JH, Kim ES, Shin SJ, et al. Development and validation of novel diagnostic criteria for intestinal Behçet's disease in Korean patients with ileocolonic ulcers. Am J Gastroenterol 2009; 104:2492-2499.

3. Dignass A, Eliakim R, Magro F, et al. Second European evidence-based consensus on the diagnosis and management of ulcerative colitis. Part 1: definitions and diagnosis (Spanish version). Rev Gastroenterol Mex 2014;79:263-289.

4. Van Assche G, Dignass A, Panes J, et al. The second European evidence-based consensus on the diagnosis and management of Crohn's disease: definitions and diagnosis. J Crohns Colitis 2010;4:7-27.

5. Chapman CG, Konda VJ. Confocal laser endomicroscopy in inflammatory bowel disease: achieving new depths in mucosal healing. Gastrointest Endosc 2016;83:792-794.

6. Cheon JH. Advances in the endoscopic assessment of inflammatory bowel diseases: cooperation between endoscopic and pathologic evaluations. J Pathol Transl Med 2015;49:209-217. 
7. Cheon JH, Kim WH. Recent advances of endoscopy in inflammatory bowel diseases. Gut Liver 2007;1:118-125.

8. Rispo A, Castiglione F, Staibano S, et al. Diagnostic accuracy of confocal laser endomicroscopy in diagnosing dysplasia in patients affected by long-standing ulcerative colitis. World J Gastrointest Endosc 2012;4:414-420.
9. Vermeire S, Van Assche G, Rutgeerts P. Classification of inflammatory bowel disease: the old and the new. Curr Opin Gastroenterol 2012;28:321-326.

10. Mowat C, Cole A, Windsor A, et al. Guidelines for the management of inflammatory bowel disease in adults. Gut 2011;60:571607. 